\title{
Metal hydride-nafion composite electrode with dual proton and electron conductivity
}

\author{
Amandeep Singh Oberoi ${ }^{*}$, John Andrews \\ School of Aerospace, Mechanical and Manufacturing Engineering, RMIT University, Melbourne, Victoria-3083, Australia
}

\begin{abstract}
A metal hydride-nafion composite electrode with dual proton and electron conductivity has been fabricated that may have applications for electrochemical storage of hydrogen. The proton conductivity of the composite electrode was measured using electrochemical impedance spectroscopy. Its electron conductivity was calculated from measurements of dc resistance. Ambient conditions were varied to study the effect of humidification and hence degree of hydration of the electrode on proton and electron conductivity of the composite electrode. The metal hydride used was a common form of AB5 alloy made from rare earth and transition element metals. Nafion-117 solution was employed as the source of nafion within the composite. Proton conductivities in the range 0.0074 to $0.0209 \mathrm{~S} / \mathrm{cm}$ were obtained, while the electron conductivities were higher, in the range 13.9 to $34.7 \mathrm{~S} / \mathrm{cm}$. It was found that the proton conductivity of the composite electrode rose with increased hydration, while electron conductivity fell.
\end{abstract}

Keywords: Metal hydride, nafion, composite electrode, proton conductivity, electron conductivity, electrochemical impedance spectroscopy

\section{Introduction}

Use of hydrogen as a future fuel is facing an important challenge of development of a safe, simple and economical energy storage system. Traditional high pressure or cryogenic hydrogen vessels both have limitations in terms of cost-effectiveness and net energy losses [1-2] and [11-15]. Absorption of hydrogen in materials like metal hydrides is a promising method of storage of hydrogen but gravimetric densities of less than $2 \mathrm{wt} \%$ have been so far obtained.

Andrews and Seif Mohammadi (2013) have recently proposed the novel concept of a 'proton flow battery' based on a reversible proton exchange membrane (PEM) fuel cell with an integrated solid-state electrode capable of storing hydrogen in atomic form. Some preliminary results on the performance of an experimental proton flow battery using a composite metal hydride - nafion electrode for storing hydrogen are reported by these authors.

For efficient performance it is important for the composite electrode to have dual proton conductivity and electron conductivity within the optimum values. The present paper therefore describes a special method to measure the proton conductivity of a composite MH-nafion electrode using Electrochemical Impedance Spectroscopy. The electron conductivity of the same electrode is also measured. The effects on these conductivities of the level of hydration of the $\mathrm{MH}-\mathrm{Nafion}$ composite are investigated.

\section{Experimental}

The equipment and materials used in the experimental measurement of the proton and electron conductivities are presented in Table 1 .

\footnotetext{
* Manuscript received July 5, 2013; revised October 10, 2013.

Corresponding author. Tel.: +61 434485 514; E-mail address: s3364207@ student.rmit.edu.au.
} 
Table 1. List of equipment and materials used in this research

\begin{tabular}{clll}
\hline \hline \multirow{2}{*}{ Sr. No. } & \multicolumn{2}{c|}{ Equipment and materials } \\
\cline { 2 - 4 } & Equipment/materials & Manufacturer & Model/registered product name \\
\hline 1 & Metal hydride powder & Sigma-Aldrich & $\mathrm{Ab}_{5}$ \\
2 & Ion exchange membrane & Sigma-Aldrich & Nafion®117 \\
3 & Nafion solution & Alfa Aesar & Perfluorosulfonic Acid \\
4 & Electrochemical interface & CH instruments & EIS workstation \\
5 & Humidity chamber & Thermoline scientific & TCH-150-SD \\
6 & Oven & Thermoline scientific & TEO-24G \\
\hline \hline
\end{tabular}

Table 2. Specifications of the prepared samples

\begin{tabular}{cccccc}
\hline \hline Sample & Condition & Width of Sample $W(\mathrm{~cm})$ & Length of Sample $L(\mathrm{~cm})$ & Area $A=W \times L\left(\mathrm{~cm}^{2}\right)$ & Thickness $(\mathrm{cm})$ \\
\hline MHN-RC & Room Condition & 0.197 & 0.197 & 0.038809 & 0.074 \\
MHN-RH75 & RH - 75\% & 0.199 & 0.198 & 0.039402 & 0.074 \\
MHN-WC & Wet condition & 0.186 & 0.185 & 0.03441 & 0.074 \\
\hline \hline
\end{tabular}

$\mathrm{An} \mathrm{AB}_{5}$ alloy metal hydride mixed with nafion-117 solution (perfluorosulfonic acid) was chosen as the materials to fabricate the composite MH-nafion composite electrode. The MH-nafion solution was stirred well before pouring it into a mould to attain the desired shape after evaporation of the solvent in which the nafion ionomer was dissolved. The mould containing this mixture was kept at room temperature for 12 hours, and then heated in an electric oven at $110^{\circ} \mathrm{C}$ for two hours, to evaporate the solvent and form the solid electrode.

The MH-nafion composite electrodes obtained from the mould were exposed to atmospheres with different relative humidities to hydrate the electrodes to various levels. Three samples, named as MHNRC, MHN-RH75, and MHN-WC with specifications shown in Table 2, were prepared. Sample MHN-RC was stored in room conditions (relative humidity around 60\%) for 24 hours. Sample MHN-RH75 was stored in a humidity chamber maintained at $75 \%$ relative humidity. Sample MHN-WC was stored in deionized water for 24 hours before testing. All samples were then subjected to electrochemical impedance spectroscopy to measure their proton conductivities.

To measure its proton conductivity, each sample was sandwiched between two nafion-117 membranes backed by two gold-plated metal electrodes in, as shown in Fig. 1. The usage of the two nafion membranes on either side of the composite electrode ensured there was no direct electrical (that is, electron) current between the two electrodes of the EIS instrument, given that the composite electrode is itself a good electron conductor.

The two nafion membranes used in the sandwich were made from a single piece of nafion-117 membrane treated with $\mathrm{H}_{2} \mathrm{SO}_{4}$ and boiled in de-ionized water to remove dirt, grease and stains and hence to obtain a clear membrane for testing. The cleaned membrane was cut in two pieces to match the size of the gold-plated metal electrodes which are to be used in the EIS technique. Two gold-plated metal electrodes were cleaned with acetone and ethanol in order to remove any accumulated dirt or grease.

The positive and negative leads of the EIS workstation were applied to the overall test assembly (Fig. 1). Nyquist plots, i.e. $Z$ ' vs $-Z^{\prime}$, were obtained for frequencies varying from $0.1 \mathrm{~Hz}$ to $10^{6} \mathrm{~Hz}$.

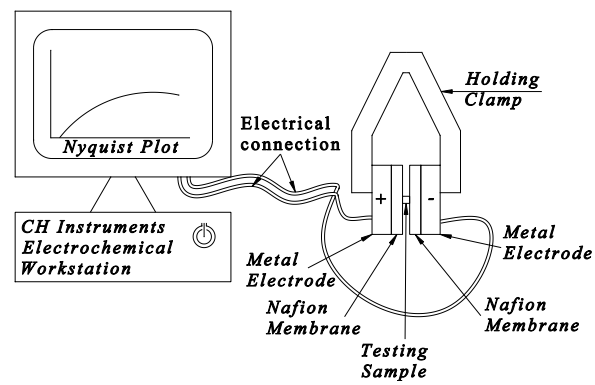

Fig. 1. Measurement of proton resistance of the samples using Electrochemical Impedance Spectroscopy technique. 
Proton resistances obtained from the EIS technique, as the high-frequency intercepts on the real impedance $\left(Z^{\prime}\right)$ axis, were used to calculate proton conductivity of the sample along with their cross sectional areas and thicknesses. The tests were repeated with all samples i.e. MHN-RC, MHN-RH75 and MHN-WC, and hence their respective proton conductivities were calculated.

A standard ohm-meter was employed to measure electron resistance using outer electrodes that had the same cross sectional area as the sample electrodes. Hence electron conductivity for all three different samples was calculated using the known dimensions of the electrodes.

\section{Results and Discussions}

When the AC voltage is applied through the electrochemical workstation in the frequency range of 0.1 to $10^{6} \mathrm{~Hz}$, a Nyquist plot is obtained as shown in Fig. 2 for the MHN-RC electrode. The intercept of the Nyquist plot on the higher frequency end of the curve is a measure of the total series resistance (real component of the total impedance). This intercept can best be estimated from the zoomed view of the high frequency end of the Nyquist, as shown in Fig. 3. The proton resistances of the nafion membranes were measured separately using EIS. The measured proton resistances of nafion membranes and resistances of the gold-plated metal electrodes employed in the setup were subtracted from the total proton resistance of the whole setup to obtain the proton resistance of the sample.

The Nyquist plot and zoom view for the MHN-RH75 sample are shown respectively in Fig. 4 and Fig. 5, and the corresponding plots for the MHN-WC electrode are given in Fig. 6 and Fig. 7.

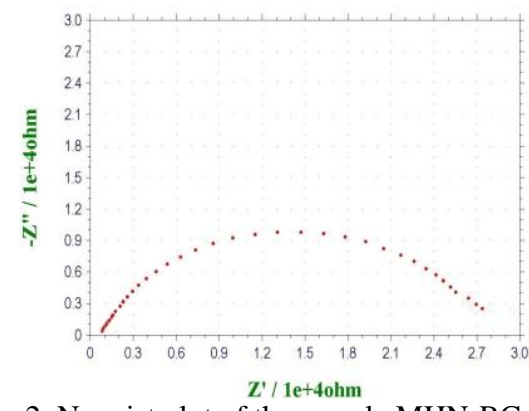

Fig. 2. Nyquist plot of the sample MHN-RC.

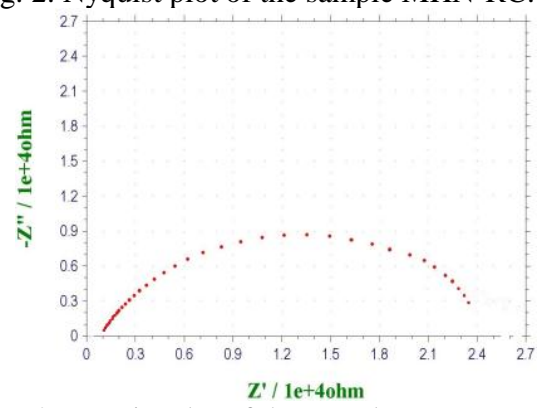

Fig. 4. Nyquist plot of the sample MHN-RH75.

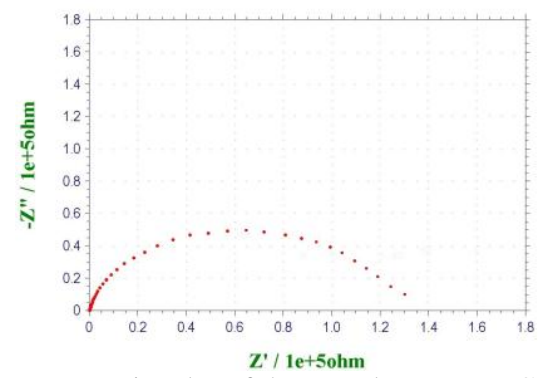

Fig. 6. Nyquist plot of the sample MHN-WC.

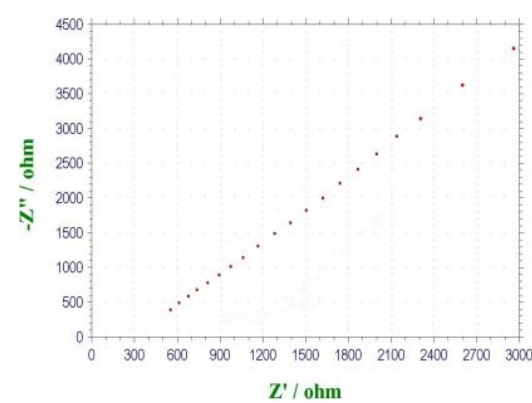

Fig. 3. Zoomed view of Nyquist plot of sample MHN-RC.

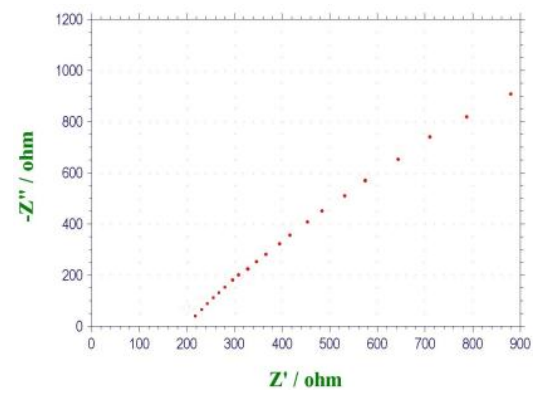

Fig. 5. Zoomed view of Nyquist plot of sample MHN-RH75.

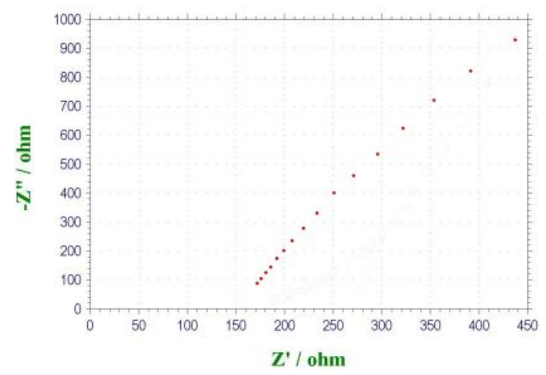

Fig. 7. Zoomed view of Nyquist plot of sample MHN-RH75. 
Table 3. Proton and electron conductivities of the samples

\begin{tabular}{lcccc|}
\hline \hline Sample & $\begin{array}{c}\text { Proton Resistance of } \\
\text { the sample }(\Omega)\end{array}$ & $\begin{array}{c}\text { Electrical Resistance } \\
\text { of the sample }(\Omega)\end{array}$ & $\begin{array}{c}\text { Proton conductivity } \\
\text { of the sample }(\mathrm{S} / \mathrm{cm})\end{array}$ & $\begin{array}{c}\text { Electrical conductivity of } \\
\text { the sample }(\mathrm{S} / \mathrm{cm})\end{array}$ \\
\hline MHN-RC & 300 & 0.055 & 0.0074 & 34.7 \\
MHN-RH75 & 200 & 0.078 & 0.0118 & 24.1 \\
MHN-WC & 150 & 0.155 & 0.0209 & 13.9 \\
\hline \hline
\end{tabular}

The proton resistances for the three samples at differing levels of hydration obtained by this method are presented in Table 3. The corresponding proton conductivities of the electrodes also given in Table 3 were calculated from their known cross sectional areas and thicknesses using the following equation:

$$
\sigma_{p}=t / A R
$$

where $\sigma_{p}=$ proton conductivity of the sample, $t=$ thickness of the sample, $A=$ area of the sample, and $R=$ proton resistance of the sample.

At room condition the proton conductivity of the electrode was found to be less (equal to $0.0074 \mathrm{~S} / \mathrm{cm}$ ) as compared to the electron conductivity (equal to $34.7 \mathrm{~S} / \mathrm{cm}$ ). As the level of hydration of the electrode goes up at relative humidity of $75 \%$, the proton conductivity rises (equal to $0.0118 \mathrm{~S} / \mathrm{cm}$ ) and the electron conductivity gets lower (equal to $24.1 \mathrm{~S} / \mathrm{cm}$ ) as compared to the respective conductivities at room conditions $(0.0074 \mathrm{~S} / \mathrm{cm}$ and $34.7 \mathrm{~S} / \mathrm{cm})$. Under wet conditions, the proton conductivity further rises up (equal to $0.0209 \mathrm{~S} / \mathrm{cm}$ ) and electron conductivity gets further down (equal to $13.9 \mathrm{~S} / \mathrm{cm}$ ).

The electron resistances directly across each electrode measured using a standard ohm meter are also given in Table 3, along with the corresponding calculated electron conductivities. The proton conductivities of the three samples are compared in Fig. 8, and the electron conductivities compared in Fig. 9.

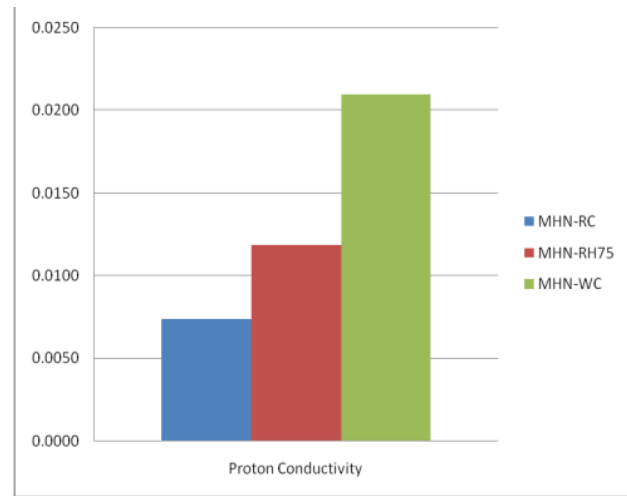

Fig. 8. The proton conductivity $(\mathrm{S} / \mathrm{cm})$ for the three different sample composite electrodes: MHN-RC at ambient relative humidity; MHN-RH75 at 75\% relative humidity; MHN-WC at full hydration.

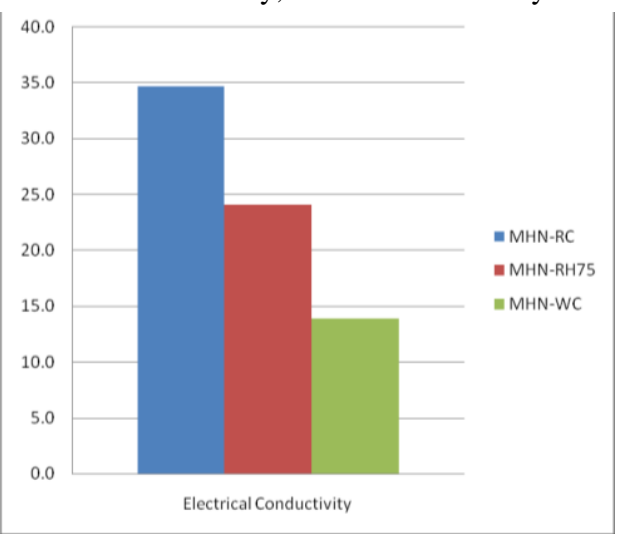

Fig. 9. Graph showing variation in proton conductivity for the three different samples. 


\section{Conclusion}

The proton conductivity of the composite MH-nafion electrode rose 0.0074 to $0.0209 \mathrm{~S} / \mathrm{cm}$ with increasing hydration. The measured electron conductivities were much higher, in the range 13.9 to 34.7 $\mathrm{S} / \mathrm{cm}$, and were found to fall with increasing hydration. The increase in proton conductivity with more water content is consistent with the usual behaviour of nafion. A possible explanation of the fall in electron conductivity with increasing hydration is that nafion swells as its water content increases, so that the area of contact between the MH particles embedded in the nafion is likely to be diminished.

Importantly for their potential application in a proton flow battery and in other areas, the composite MH-nafion electrode made and tested in this study has shown acceptable levels of both proton and electron conductivity.

\section{References}

[1] Andrews J. and Seif Mohammadi S., Towards a 'proton flow battery': Investigation of a reversible PEM fuel cell with integrated metal-hydride hydrogen storage. International Journal of Hydrogen Energy, submitted for publication, 2013.

[2] Andrews J. and Doddathimmaiah A, Regenerative fuel cells. in Fuel Cell Materials, M. Gasik, Ed., Finland: Woodhead Publishing, 2008, pp. 344-385.

[3] Beguin F., Friebe M., Jurewicz K. et al., State of hydrogen electrochemically stored using nanoporous carbons as negative electrode materials in an aqueous medium. Science Driect-Carbon 44, 2392-2398, 2006.

[4] Blenda-Martinez M.J., Perez J.M. et al., Effect of surface chemistry on electrochemical storage of hydrogen in porous carbon materials. Science Driect - Carbon 46, 1053-1059, 2008.

[5] Frackowiak E. and Beguin F., Electrochemical storage of energy in carbon nano-tubes and nano-structured carbon. Science Direct-Carbon 40, 1775-1787, 2002.

[6] Guterl C., Frackowiak E., Jurewicz K., et al., Electrochemical energy storage in ordered porous carbon materials. Science Direct-Carbon 43, 1293-1302, 2005.

[7] Hynek S., Fuller W., Bentley J., Hydrogen storage by carbon sorption. International Journal of Hydrogen Energy, 22: 601-610, 1997.

[8] Latroche M., Structural and thermodynamic properties of metallic hybrids used for energy storage. Journal of Phys Chem Solids, 65: $517-522,2004$.

[9] Liu N. N., Yin L. W. et al., Enhanced electrochemical hydrogen storage capacity of activated mesoporous carbon materials containing nickel inclusions. International Journal of Hydrogen Energy, 35: 12410-12420, 2010.

[10] Reunchan P. and Jhi S. H., Metal-dispersed porous graphene for hydrogen storage. Applied Physics Letter, 2011, 98: 093103-3.

[11] Ma S. H., Akiko K., Zyun S., and Kazuaki Y., Measuring conductivity of proton conductive membranes in the direction of thickness. Espec Technology Report, 20: 12-20.

[12] Sakintuna B., Lamari-Darkrim F., and Hirscher M, Metal hydride materials for solid hydrogen storage: A review. International Journal of Hydrogen Energy, 32: 1121-1140.

[13] Shinnar R., The hydrogen economy, fuel cells, and electric cars. International Journal of Technology in Society, 25: 45-476.

[14] Yu Y. M., Zhao N. Q., Shi C. S., et al., Electrochemical hydrogen storage of expanded graphite decorated with $\mathrm{TiO}_{2}$ nanoparticles. International Journal of Hydrogen Energy, 30:1-7, 2012.

[15] Y. Sone, et al., Ionic conductivity of an extrude nafion. Jornal of the Electrochemical Society, 143: 1254, 1996. 